\title{
Light trapping in solar cells: When does a Lambertian scatterer scatter Lambertianly?
}

\author{
Corsin Battaglia, ${ }^{\text {a) }}$ Mathieu Boccard, Franz-Josef Haug, and Christophe Ballif \\ Ecole Polytechnique Fédérale de Lausanne (EPFL), Institute of Microengineering (IMT), \\ Photovoltaics and Thin Film Electronics Laboratory, 2000 Neuchâtel, Switzerland
}

(Received 26 June 2012; accepted 3 October 2012; published online 5 November 2012)

\begin{abstract}
We derive scaling laws for the Rayleigh-Sommerfeld formulation we recently developed to describe light scattering from nanotextured interfaces. These scaling laws provide precious intuition on how to link scattering from different interfaces. In particular, we answer the question how to obtain a Lambertian scatterer into silicon, starting from a Lambertian scatterer into air relevant to the development of light trapping schemes in thin-film silicon solar cells. We also define a Lambertionality factor which measures how close an arbitrary scatterer approaches Lambertian scattering and extend the fundamental $4 n^{2}$ light trapping limit to arbitrary scattering distributions. (C) 2012 American Institute of Physics. [http://dx.doi.org/10.1063/1.4761988]
\end{abstract}

\section{INTRODUCTION}

Light scattering has gained tremendous importance in the field of photovoltaics, as absorption of sunlight in solar cells can be enhanced drastically by proper engineering of photonic nanostructures. Assuming a Lambertian scatterer, theory predicts an upper limit of $4 n^{2}$ for the absorption enhancement inside a weakly absorbing planar optical absorber of refractive index $n .{ }^{1}$ In particular, for thin-film silicon solar cells, advanced light trapping schemes are crucial to boost conversion efficiency, as the absorption coefficient of silicon becomes small towards the near infrared region.

Significant progress has been made in recent years in developing experimental methods for implementing arbitrary scattering morphologies into high-efficiency thin-film silicon devices. ${ }^{2-4}$ From a theoretical point of view, the rigorous numerical solution of Maxwell's equations has become feasible for a limited number of simple morphologies, ${ }^{5-8}$ but often becomes impractical for more complex morphologies, as it requires massive computational resources.

Some of us recently presented a theoretical framework ${ }^{9}$ based on a slightly modified Rayleigh-Sommerfeld diffraction integral proposed by Harvey, ${ }^{10}$ for the calculation of the angular and spectral dependence of light scattered at a nanotextured interface. Our scalar approach requires only measured profile data and the refractive indices of the interface as input and can be solved efficiently within seconds. The model was validated in a variety of experimental configurations ${ }^{9,11-15}$ and compared positively to other scalar and rigorous treatments. ${ }^{16,17}$

Here, we derive analytic scaling laws from our theory which link scattering from different interfaces. From these scaling laws, one can easily understand why a nanotextured surface with a Lambertian scattering profile into air does not scatter in a Lambertian fashion into silicon. More importantly, our scaling laws tell us what is needed to obtain a Lambertian scatterer into silicon. Furthermore, we define a Lambertionality factor that represents a useful and physically

${ }^{a}$ Electronic mail: corsin.battaglia@epfl.ch. URL: http://pvlab.epfl.ch/. meaningful measure of how close an arbitrary scatterer approaches Lambertian scattering and discuss its relation to the $4 n^{2}$ limit.

\section{SCATTERING MODEL}

For the sake of completeness, we present again our scattering model introducing a more explicit formulation then in Ref. 9. Light passing across a nanotextured interface, with a peak-to-valley roughness of $z_{0}$, is assumed to acquire a phaseshift proportional to $n_{1} \cdot z+n_{2} \cdot\left(z_{0}-z\right)$, where $z$ is the distance from the maximum peak height to the interface traveled in the first medium with refractive index $n_{1}$, and $\left(z_{0}-z\right)$ the distance traveled after the interface in the second medium with refractive index $n_{2}$ (see Fig. 1). Since the interface is textured, $z$ depends on the morphology of the surface and is therefore a function of the lateral coordinates $x$ and $y$. Thus, the local phaseshift acquired by a plane wave after passing the interface is $\left(n_{1}-n_{2}\right) \cdot z(x, y)+n_{2} \cdot z_{0}$. Summing up all plane waves exiting the roughness zone, taking into account this phaseshift, we obtain the radiance $L$ in direction cosine space for a given wavelength $\lambda$

$$
\begin{aligned}
L(\alpha, \beta, \lambda)= & c(\lambda) \mid \iint d x \cdot d y \cdot e^{2 \cdot \pi \cdot i \cdot \frac{n_{2}}{\lambda} \cdot(\alpha \cdot x+\beta \cdot y)} \\
& \times\left. e^{2 \cdot \pi \cdot i \cdot \frac{\left|n_{1}-n_{2}\right|}{\lambda} \cdot z(x, y)}\right|^{2} .
\end{aligned}
$$

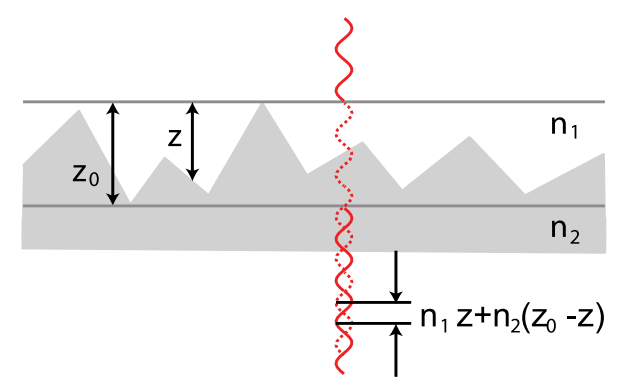

FIG. 1. A plane wave passing through the roughness zone acquires a phase shift. 
Note that the term $n_{2} \cdot z_{0}$ in the phaseshift leads to a position independent phase which drops out when taking the absolute value for the radiance. $c(\lambda)$ is a wavelength dependent normalization constant. Here, $\alpha$ and $\beta$ are direction cosines in reciprocal space, related to the wavevector $\mathbf{k}$ via $\mathbf{k}=\frac{2 \cdot \pi}{\lambda}$ $\left(\alpha, \beta, \sqrt{1-\alpha^{2}-\beta^{2}}\right)$. The double integral can be handled efficiently using a fast Fourier transform approach.

For comparison of the angular dependence with experiment, it is convenient to transform the radiance from direction cosine space $(\alpha, \beta)$ into projected (unit radius) spherical coordinates $(\theta, \phi)$, using $\alpha=\sin \theta \cdot \cos \phi$ and $\beta=\sin \theta \cdot \sin \phi$. The angle resolved scattering (ARS) curve for a given wavelength $\lambda$ is obtained by taking into account the projection factor $\cos \theta=\sqrt{1-\alpha^{2}-\beta^{2}}$

$$
\operatorname{ARS}(\theta, \phi, \lambda)=L(\theta, \phi, \lambda) \cdot \cos \theta .
$$

When designing light trapping in solar cells with random scatterers that do not show any azimuthal dependence $\phi$ at normal incidence, it is often more useful to consider the azimuthally integrated ARS, labeled $\mathrm{ARS}_{\bar{\phi}}$, as it reflects the total amount of intensity scattered into a given polar angle $\theta$

$$
\operatorname{ARS}_{\bar{\phi}}(\theta, \lambda)=\int_{0}^{2 \pi} d \phi \cdot \sin \theta \cdot \cos \theta \cdot L(\theta, \phi, \lambda) .
$$

Here, $\sin \theta$ represents the Jacobian of the integration in spherical coordinates. A pragmatic definition for the normalization factor $c(\lambda)$ proposed by Harvey ${ }^{10}$ is obtained by requiring that

$$
\int_{0}^{2 \pi} d \phi \int_{0}^{\pi / 2} d \theta \cdot \sin \theta \cdot \cos \theta \cdot L(\theta, \phi, \lambda)=1,
$$

which guarantees that all propagating modes sum up to 1 , i.e., to the total amount of transmitted light. This approximation is appropriate as long as the amount of light that is scattered into evanescent modes remains negligible. It is applicable to the case of weakly absorbing media, such as a rough $\mathrm{ZnO}$-air interface, but fails for a rough silver-air interface, where surface plasmon polaritons may cause strong absorption. ${ }^{18}$

With this definition, the spectral dependence of the scattered light as measured by haze $H$ may directly be obtained from the radiance via

$$
H(\lambda)=\int_{0}^{2 \pi} d \phi \int_{>0}^{\pi / 2} d \theta \cdot \sin \theta \cdot \cos \theta \cdot L(\theta, \phi, \lambda) .
$$

Therefore, knowing the radiance $L$, we can determine both ARS and haze.

\section{LAMBERTIAN SCATTERING}

For an ideal Lambertian scatterer which exhibits an isotropic radiance, $L=1 / \pi$. This definition satisfies the normalization in Eq. (2). Consequently, $\operatorname{ARS}(\theta, \phi)$ has a simple $\cos \theta$ dependency and $\operatorname{ARS}_{\bar{\phi}}(\theta)$ as shown in Fig. 2(b) is proportional to $\sin \theta \cos \theta$. For an arbitrary scatterer, the radiance at a given wavelength is completely defined by the refractive indices $n_{1}$ and $n_{2}$ and the surface profile $z(x, y)$ of

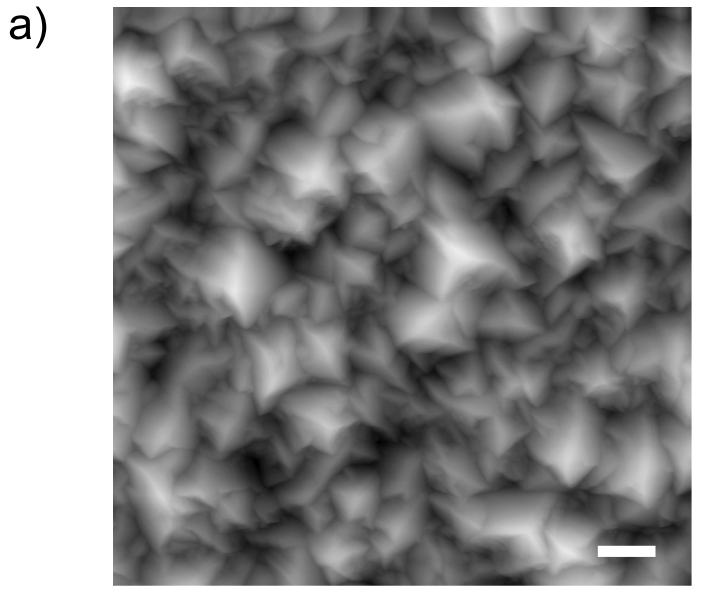

b)

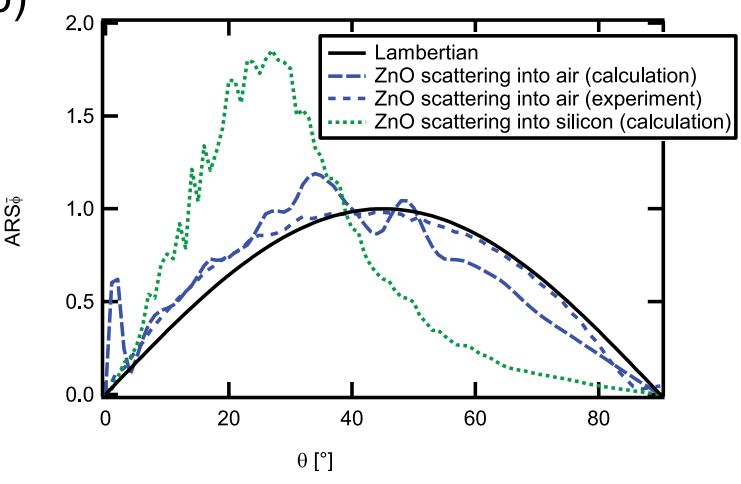

FIG. 2. (a) AFM image of $\mathrm{ZnO}$ grown by low-pressure chemical vapor deposition. ${ }^{33}$ Scale bar $1 \mu \mathrm{m}$. (b) $\mathrm{ARS}_{\bar{\phi}}$ for an ideal Lambertian scatterer (black curve), the $\mathrm{ZnO}$ morphology for scattering into air (blue curves), and silicon (green curve) at $\lambda=543 \mathrm{~nm}$. The dashed blue curve was measured experimentally.

the interface which can be determined experimentally for example by atomic force microscopy (AFM). Thin-film silicon solar cells (in $p-i-n$ configuration) typically consist of a glass substrate $(n \approx 1.5)$, a transparent conductive oxide (TCO) layer $(n \approx 2)$ acting as front electrode, the silicon layers $(n \approx 4)$ and a back electrode. Light scattering is achieved at the TCO-silicon interface by texturing the TCO surface. A characteristic TCO surface morphology with a root mean square roughness of $200 \mathrm{~nm}$ and a correlation length of $400 \mathrm{~nm}$ used for light trapping in thin-film silicon modules is shown in Fig. 2(a). In Fig. 2(b), we show the experimentally determined $\mathrm{ARS}_{\bar{\phi}}$ for this TCO for scattering into air at $\lambda=543 \mathrm{~nm}$. The haze at this wavelength is equal to 1 . From comparison with the corresponding curve for the ideal Lambertian scatterer, we conclude that light scattering from this TCO into air is very close to Lambertian. Using the theoretical expression in Eq. (1) for the radiance, we can also calculate the $\mathrm{ARS}_{\bar{\phi}}$ from the AFM image. The calculated $\mathrm{ARS}_{\bar{\phi}}$ curve reproduces the experimental $\mathrm{ARS}_{\bar{\phi}}$ curve well. We note that it deviates slightly at large scattering angles because we model the optical interface by a random phasescreen with zero thickness which neglects diffraction within the peak-to-valley depth of the rough interface. ${ }^{19}$ The oscillations in the calculation are due to the finite AFM image size and may be reduced by either taking larger images or averaging over several images. ${ }^{20}$ 
The normalization in Eq. (2) should be applied with care to cases where the haze drops below 1 as the Jacobian $\sin \theta$ gives zero weight to the specular beam $(\theta=0)$. In this case, $\operatorname{ARS}_{\bar{\phi}}(\theta)$ can still take its maximum at $\theta=45^{\circ}$, but its maximum will be below 1 , which reflects the fact that a certain fraction of light is not scattered at all, but remains in the specular beam. However, the scattered fraction of light will exhibit an angular dependence identical to a Lambertian scatterer.

Although not measurable experimentally (see however Ref. 16), we can calculate the $\mathrm{ARS}_{\bar{\phi}}$ for scattering into silicon using our scattering algorithm. The resulting curve, also given in Fig. 2(b) at $\lambda=543 \mathrm{~nm}$ where haze equals 1 , shows that the scattering profile is far from Lambertian. Therefore, a Lambertian scatterer into air is not necessarily a Lambertian scatterer into silicon.

\section{SCALING LAWS}

But then, what is needed to obtain a Lambertian scatterer into silicon? Inspection of Eq. (1) shows that the input parameters $n_{1}, n_{2}, \lambda$, and $z(x, y)$ enter the radiance via the three terms

$$
\frac{n_{2} \cdot x}{\lambda}, \quad \frac{n_{2} \cdot y}{\lambda} \text { and } \frac{\left|n_{1}-n_{2}\right| \cdot z}{\lambda} .
$$

Keeping these three terms constant will keep the radiance invariant.

Different sets of parameters satisfying this conditions are listed in Table I. Set A of parameters represents scattering from a nanotextured TCO $\left(n_{1}=2\right)$ into air $\left(n_{1}=1\right)$. This is the case, we discussed above for which ARS and haze can be measured experimentally. Set B of parameters represents scattering from the TCO $\left(n_{1}=2\right)$ into silicon $\left(n_{2}=4\right)$. To obtain the same values for the parameters in Eq. (3), we have to scale $\lambda$ by a factor of 4 and the structure heights given by $z$ by a factor of 2 . This means that if we increase the aspect ratio of the structure by a factor of 2 and go to 4 times the wavelength, we get the same ARS for scattering into silicon as for scattering into air. Consequently if scattering into air is Lambertian, as is almost the case for the TCO in Fig. 2(a), we now have a way, to determine the corresponding structure which gives Lambertian scattering into silicon.

More generally, to go from an arbitrary interface characterized by the refractive indices $n_{1}$ and $n_{2}$ to another interface with $n_{1}^{\prime}$ and $n_{2}^{\prime}$, we can define a scaled wavelength $\lambda^{\prime}$ and a scaled structure height $z^{\prime}$

$$
\lambda^{\prime}=\frac{n_{2}^{\prime}}{n_{2}} \cdot \lambda, \quad \text { and } \quad z^{\prime}=\frac{n_{2}^{\prime} \cdot\left|n_{1}-n_{2}\right|}{n_{2} \cdot\left|n_{1}^{\prime}-n_{2}^{\prime}\right|} \cdot z
$$

TABLE I. Set of parameters resulting in the same radiance.

\begin{tabular}{cccccccccc}
\hline \hline Set & $n_{1}$ & $n_{2}$ & $\lambda$ & $x$ & $y$ & $z$ & $\frac{n_{2} \cdot x}{\lambda}$ & $\frac{n_{2} \cdot y}{\lambda}$ & $\frac{\left|n_{1}-n_{2}\right| \cdot z}{\lambda}$ \\
\hline A & 2 & 1 & $\lambda_{0}$ & $x_{0}$ & $y_{0}$ & $z_{0}$ & $\frac{x_{0}}{\lambda_{0}}$ & $\frac{y_{0}}{\lambda_{0}}$ & $\frac{z_{0}}{\lambda_{0}}$ \\
B & 2 & 4 & $4 \cdot \lambda_{0}$ & $x_{0}$ & $y_{0}$ & $2 \cdot z_{0}$ & $\frac{x_{0}}{\lambda_{0}}$ & $\frac{y_{0}}{\lambda_{0}}$ & $\frac{z_{0}}{\lambda_{0}}$ \\
C & 2 & 4 & $\lambda_{0}$ & $\frac{x_{0}}{4}$ & $\frac{y_{0}}{4}$ & $\frac{z_{0}}{2}$ & $\frac{x_{0}}{\lambda_{0}}$ & $\frac{y_{0}}{\lambda_{0}}$ & $\frac{z_{0}}{\lambda_{0}}$ \\
\hline \hline
\end{tabular}

so that the radiance, and with it ARS and haze remain invariant when we replace $\lambda$ by $\lambda^{\prime}$ and $z$ by $z^{\prime}$.

Within scalar scattering theory as described for example in Ref. 21, scattering from TCOs into air can be linked to scattering from TCOs into silicon by going to twice the wavelength as haze depends only on the fraction $\left|n_{1}-n_{2}\right|$. $z / \lambda$ (Ref. 22). This correctly reproduces the phaseshift in Eq. (1) and therefore the amount of light that is scattered away from the specular beam, i.e., the haze. However, by making this approximation, one neglects light refraction described by the lateral scaling by $n_{2}$ introduced in Eq. (1) via the terms $n_{2} \cdot x / \lambda$ and $n_{2} \cdot y / \lambda$ so that the angular scattering distribution, which is crucial for light trapping in solar cells, is not correctly reproduced.

Set $\mathrm{C}$ of parameters provides a complementary prescription how to link scattering into air to scattering into silicon, with the advantage of working at the same wavelength. In this case, the heights $z$ have to be scaled by a factor $1 / 2$, while the lateral dimensions $x$ and $y$ must be scaled by a factor of $1 / 4$. Note that in terms of aspect ratio, we also obtain a scaling of 2 as before, while all space coordinates are inversely scaled by the factor of 4 . So according to the second prescription, we obtain a Lambertian scatterer into silicon, by reducing the feature size by a factor of 4 and by increasing the aspect ratio by a factor of 2 .

More generally, to go from an arbitrary interface characterized by the refractive indices $n_{1}$ and $n_{2}$ to another interface with $n_{1}^{\prime}$ and $n_{2}^{\prime}$, we can define a set of scaled coordinates $x^{\prime}, y^{\prime}$, and $z^{\prime}$

$$
x^{\prime}=\frac{n_{2}}{n_{2}} \cdot x, \quad y^{\prime}=\frac{n_{2}}{n_{2}^{\prime}} \cdot y, \quad \text { and } \quad z^{\prime}=\frac{\left|n_{1}-n_{2}\right|}{\left|n_{1}^{\prime}-n_{2}^{\prime}\right|} \cdot z,
$$

so that the radiance, and with it also ARS and haze, are invariant.

In the considerations above, we neglected the dispersion of the experimental refractive indices with wavelength. One can account for the dispersion by making the scaling coefficients wavelength dependent. This of course leads to the inconvenience that the scaling of the structure becomes wavelength dependent. However, in the wavelength range in which light scattering is important for thin-film silicon solar cells, the refractive indices can be assumed constant.

We emphasize that the importance of these scaling laws is that they provide an intuitive prescription how to obtain a desired scattering profile, when going from one interface to another. To improve light scattering of the TCO morphology in Fig. 1(a) into silicon towards an ideal Lambertian scatterer, we need to increase the aspect ratio. A similar conclusion was derived by Fahr et al. ${ }^{23}$ from numerical calculations within the much more complicated framework of rigorous coupled wave analysis (RCWA). Although an increased aspect ratio is beneficial for the optical cell performance, we stress that this approach is usually detrimental for the electrical cell performance. ${ }^{4,24-26}$ So a trade-off must be found. However, the scaling relations also tell us that features for scattering into silicon can be four times smaller than for scattering into air. Even if this requires a higher aspect ratio, we recently showed that small sharp features harm the electrical cell performance 
much less than large features. ${ }^{27,28}$ Our observations therefore provide a clear roadmap for integration of such structures into devices. Although nature does not allow the growth of $\mathrm{ZnO}$ morphologies with arbitrary aspect ratio, ${ }^{12}$ a scaled $\mathrm{ZnO}$ morphology, for quasi-Lambertian scattering into silicon, could be fabricated using our recently developed nanomoulding technique. $^{3}$

\section{LAMBERTIONALITY}

To measure how close an arbitrary scatterer approaches Lambertian scattering, we define the Lambertionality factor $a(\lambda)$

$$
a(\lambda)=\int_{0}^{2 \pi} d \phi \int_{0}^{\pi / 2} d \theta \cdot \sin \theta \cdot \frac{1}{\cos \theta} \cdot A R S(\theta, \phi, \lambda),
$$

$a(\lambda)$ represents the average light path length enhancement of light rays obtained from a given scattering distribution. Here the factor $1 / \cos \theta$ takes into account the path length enhancement of an individual light ray which is scattered into an angle $\theta$ (see Fig. 3(a)). The contribution of each light ray is weighted by $\operatorname{ARS}(\theta, \phi, \lambda)$. For an ideal Lambertian scatterer $a=2(\operatorname{ARS}=\cos \theta / \pi)$, whereas for a flat specular interface that does not scatter light at all $a=1(\operatorname{ARS}=\delta(\theta) \cdot \delta(\phi) /$ $\sin \theta$, where $\sin \theta$ is an integral part of the Dirac $\delta$ in spherical coordinates). The Lambertionality factor can exceed values of 2 in a limited wavelength range, for instance, using a grating with suppressed zero diffraction order which scatters all light into an angle higher than $\theta=60^{\circ}$ (which corresponds to $a=2$ ), resulting in super-Lambertian scattering. Fig. 3(b) shows the Lambertionality factor for the $\mathrm{ZnO}$ morphology for scattering into air and scattering into silicon. For scattering into air at short wavelengths, the $\mathrm{ZnO}$ scatters almost like a Lambertian. For longer wavelengths, the scattering profile deviates more and more from the Lambertian profile. For scattering into silicon, light scattering is clearly sub-Lambertian over the full wavelength range with values between 1.2 and 1.3 and a significantly weaker wavelength dependence. Note that the $1 / \cos \theta$ weighting factor diverges at large angles and therefore correctly captures the importance of large angle scattering observed in experiments. However, as our scattering algorithm slightly underestimates the scattering intensity at large angles, $a(\lambda)$ extracted from calculations has the tendency to underestimate the Lambertionality.

A second important quantity which can be derived from the radiance is the fraction of light $b(\lambda)$ which is escaping through the escape cone, i.e., is scattered into angles smaller than the critical angle $\theta_{c}$ required for total reflection

$$
b(\lambda)=\int_{0}^{2 \pi} d \phi \int_{0}^{\theta_{c}} d \theta \cdot \sin \theta \cdot A R S(\theta, \phi, \lambda),
$$

$b(\lambda)$ can be interpreted as an angle averaged value of an idealized transmission factor which is either 1 inside the escape cone and 0 outside the escape cone, although this is clearly an oversimplified description for a rough interface. Consequently, $1-b$ can be interpreted as an effective reflection coefficient. For an ideal Lambertian scatterer into air a)
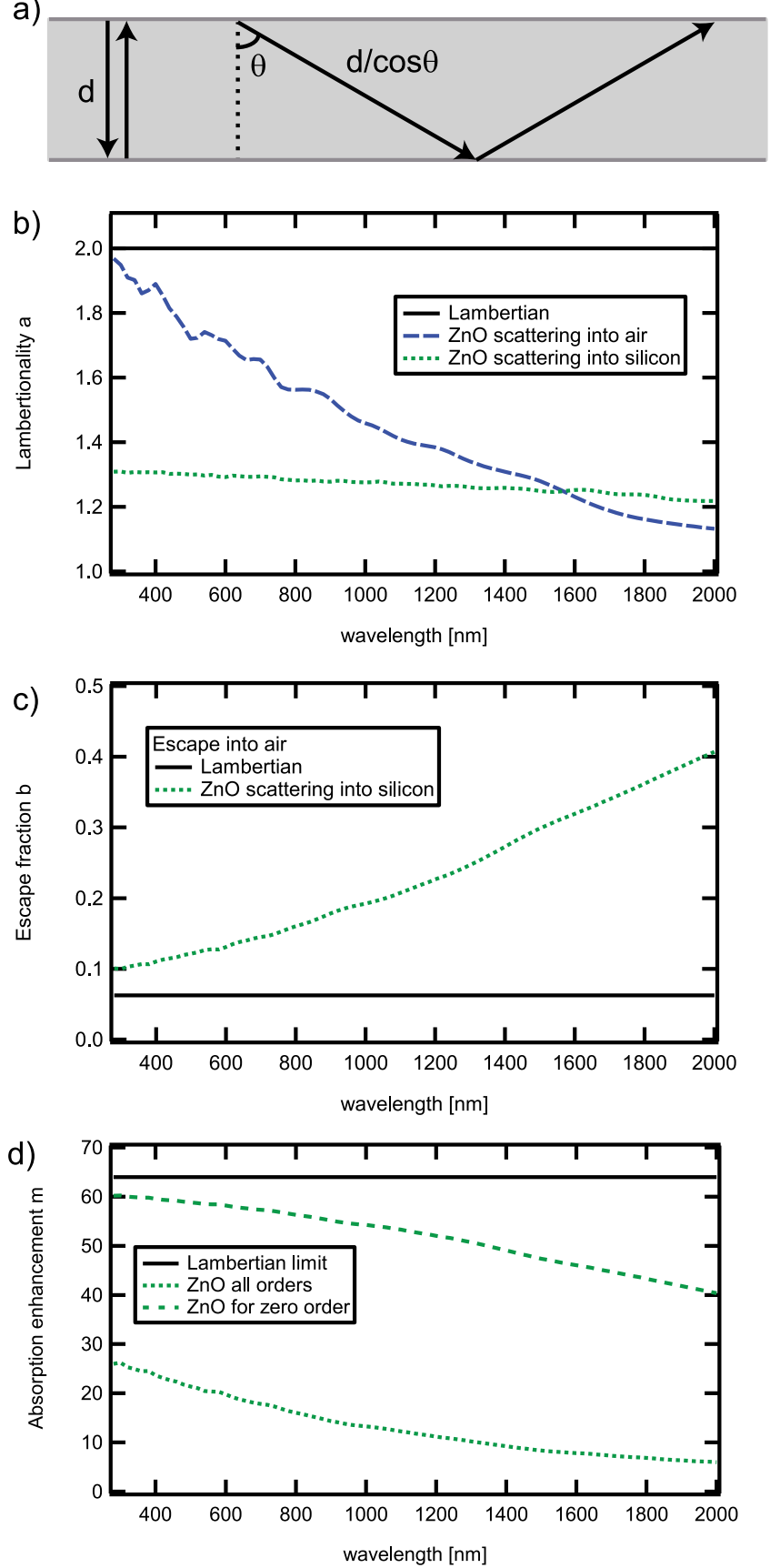

FIG. 3. (a) Sketch illustrating the physical interpretation of the Lambertionality factor. (b) Lambertionality factors $a$ for an ideal Lambertian scatterer (black curve), the $\mathrm{ZnO}$ for scattering into air (blue curve), and silicon (green curve), with the corresponding (c) escape fractions $b$ and (d) absorption enhancements $m$. "ZnO all orders" corresponds to the case described by Eq. (5), where the initial scattering distribution is used also for all subsequent scattering events. "ZnO for zero order" corresponds to the case described by Eq. (6), where only the zero order term is described by the calculated scattering distribution and all higher order scattering events are assumed to be Lambertian. The values of $m$ are only valid in the weak absorption regime. For hydrogenated amorphous silicon, this corresponds to the wavelength range between 700 and $800 \mathrm{~nm}$. For (micro-) crystalline silicon the relevant wavelength range extends from $700 \mathrm{~nm}$ to $1100 \mathrm{~nm}$.

$b=1 / n^{2}$. More generally, for Lambertian scattering from medium $n_{1}$ into a higher index medium $n_{2}, b=n_{1}^{2} / n_{2}^{2}$. Fig. 3(c) shows $1 / b$ as a function of wavelength. For a silicon absorber in air, the Lambertian scatterer scatters $6 \%$ of light into the escape cone. At short wavelengths, the $\mathrm{ZnO}$ morphology 
scatters about $10 \%$ into the escape cone, but this value is increasing rapidly with increasing wavelength.

\section{LIGHT TRAPPING LIMIT}

To establish the link between the Lambertionality factor and the $4 n^{2}$ limit, we rewrite $4 n^{2}$ as $2 \cdot 2 \cdot n^{2}$. The first factor of 2 takes into account the effect of the (specular) back reflector of the solar cell which doubles the light path. The second factor of 2 can be identified with the Lambertionality factor $a$. The factor of $n^{2}$ is simply equal to $1 / b$. To show this, we proceed similar to Deckman et al. ${ }^{29}$ and describe the absorption of the photoactive absorber layer $F$ as an infinite series of absorption round trips

$F=\left(1-e^{-2 \cdot a \cdot \cdot \cdot d}\right) \cdot(1-R) \cdot \sum_{n=0}^{\infty} e^{-2 \cdot a \cdot \alpha \cdot d \cdot n} \cdot(1-b)^{n} \cdot\left(1-A_{p}\right)^{n}$.

Here $1-e^{-2 \cdot a \cdot \cdot \cdot d}$ describes one effective round trip of the light ray in the absorber with wavelength dependent absorption coefficient $\alpha(\lambda)$ and thickness $d$ including the effect of the Lambertionality factor $a$ and the reflection at the back reflector which is taken into account by the factor of $2 . R$ accounts for light which is reflected before ever entering the absorber. Each round trip is weighted by a loss factor which takes into account the (desired) absorption in the absorber $e^{-2 \cdot a \cdot \alpha \cdot d}$, the effective reflection $1-b$ at the front interface and undesired parasitic absorption $A_{p}$ in the heavily doped layers and the electrodes which is always present in real devices and which does not contribute to current generation. Assuming ideal carrier collection which is closely satisfied in real thin-film silicon devices, $F$ can be identified with the external quantum efficiency (EQE) of the cell.

Close to the band gap of the absorber, where $\alpha \cdot d \ll 1$, and assuming no parasitic absorption $A_{p}=0$ (which can be integrated out from experimental data ${ }^{30}$ ) and no primary reflection $R=0$ (which can be achieved closely with antireflection measures), this expression simplifies to

$$
F=2 \cdot a \cdot \alpha \cdot d \cdot \sum_{n=0}^{\infty}(1-b)^{n}=2 \cdot a \cdot \alpha \cdot d \cdot \frac{1}{b} .
$$

The absorption enhancement $m$ is obtained by dividing this expression by the absorption of a single pass through the absorber $1-e^{-\alpha \cdot d}$ which reduces to $\alpha \cdot d$ in the low absorption limit. So the final enhancement factor is given simply by

$$
m=2 \cdot \frac{a}{b},
$$

which correctly reproduces the $4 n^{2}$ limit for a Lambertian scatterer with $a=2$ and $b=1 / n^{2}$. Fig. 3(d) compares the absorption enhancement obtained for the ideal Lambertian scatterer with the corresponding values for the $\mathrm{ZnO}$ morphology which turns out to be much lower than the Lambertian limit. As was already pointed out in Refs. 1 and 31, there is an overriding tendency towards full randomization after a few successive scattering events, i.e., $a$ and $b$ quickly tend towards the Lambertian values of 2 and $1 / n^{2}$, respectively. Our estimation of the absorption enhancement does not take this into account, but assumes an identical scattering distribution for every pass. More realistic values for $m$ will necessarily fall somewhere in between the Lambertian limit and the calculated value. A rudimentary but instructive way to take into account this tendency towards full randomization is by isolating the zero order term from the sum in Eq. (4) and treat the higher order terms in the sum as Lambertian

$$
m=2 \cdot a+2 \cdot a \cdot \sum_{n=1}^{\infty}(1-b)^{n} \approx 2 \cdot a+(1-b) \cdot 4 \cdot n^{2},
$$

$(1-b)$ is very important here, as it strongly damps the weight of the Lambertian $4 n^{2}$ factor. Physically this can be explained by the important fraction of light which is lost after the first non-Lambertian round trip at the first front reflection event, described by the term $(1-b)$ which is then no more available for Lambertian scattering during successive round trips. From Fig. 3(d), we see that this first order correction leads to higher values of $m$ which are closer to the Lambertian limit. Higher order corrections can be taken into account analogously, although a realistic description of how successive scattering events are approaching a Lambertian scattering profile is yet to be developed. Also the effect of a non-specular back reflector can be taken into account by attributing different values for $a$ and $b$ for forward and backward traveling light rays of successively higher order, but of course at the expense of increased mathematical complexity.

Several empirical merit criteria have been proposed to characterize the light scattering capability of a given photonic nanostructure. ${ }^{32,33}$ The advantages of the Lambertionality factor $a$ and the escape fraction $b$ as merit criteria are their intuitive physical interpretation and their analytic link to the EQE and the absorption enhancement $m$. Future work will focus on establishing the link between these two parameters extracted from AFM images and corresponding values extracted from experimental EQEs of real devices via Eq. (4) (Ref. 34).

\section{CONCLUSION}

Based on the relatively simple analytic expression for the radiance within our Rayleigh-Sommerfeld formulation, we derived useful scaling laws which relate the light scattering profiles from interfaces with different refractive indices. For a given scattering profile into air which can be measured experimentally, these scaling laws provide an intuitive prescription how to obtain an identical scattering profile into silicon which is not directly accessible by experiment. We showed that analogous scaling relations derived from the traditional scalar scattering theory correctly reproduce haze, but necessarily fail to describe the angular distribution of scattered light. We further introduced the Lambertionality factor and escape fraction as physically meaningful merit criterions for the light scattering capabilities of a given scatterer and highlighted their direct link to the fundamental light trapping limit. 


\section{ACKNOWLEDGMENTS}

We thank Didier Dominé for stimulating discussions, Peter Cuony for providing the experimental data, and acknowledge funding from the Velux Foundation and the Swiss Federal Energy Office. Part of this work was carried out in the framework of the FP7 project "Fast Track," funded by the EC under Grant Agreement No. 283501.

${ }^{1}$ E. Yablonovitch, J. Opt. Soc. Am. 72, 899 (1982).

${ }^{2}$ C. Battaglia, J. Escarré, K. Söderström, L. Erni, L. Ding, G. Bugnon, A Billet, M. Boccard, L. Barraud, S. D. Wolf, F.-J. Haug, M. Despeisse, and C. Ballif, Nano Lett. 11, 661 (2011).

${ }^{3}$ C. Battaglia, J. Escarré, K. Söderström, M. Charrière, F.-J. Haug, M. Despeisse, and C. Ballif, Nat. Photonics 5, 535 (2011).

${ }^{4}$ C. Battaglia, C.-M. Hsu, K. Söderström, J. Escarré, F.-J. Haug, M. Charrière, M. Boccard, M. Despeisse, D. T. L. Alexander, M. Cantoni, Y. Cui, and C. Ballif, ACS Nano 6, 2790 (2012).

${ }^{5}$ C. Rockstuhl, F. Lederer, K. Bittkau, T. Beckers, and R. Carius, Appl. Phys. Lett. 94, 211101 (2009).

${ }^{6}$ A. Naqavi, K. Söderström, F.-J. Haug, V. Paeder, T. Scharf, H. P. Herzig, and C. Ballif, Opt. Express 19, 128 (2011).

${ }^{7}$ R. Dewan, I. Vasilev, V. Jovanov, and D. Knipp, J. Appl. Phys. 110, 013101 (2011).

${ }^{8}$ V. E. Ferry, A. Polman, and H. A. Atwater, ACS Nano 5, 10055 (2011).

${ }^{9}$ D. Dominé, F.-J. Haug, C. Battaglia, and C. Ballif, J. Appl. Phys. 107, 044504 (2010).

${ }^{10}$ J. E. Harvey, C. L. Vernold, A. Krywonos, and P. L. Thompson, Appl. Opt. 38, 6469 (1999).

${ }^{11}$ C. Battaglia, K. Söderström, J. Escarré, F.-J. Haug, D. Dominé, P. Cuony, M. Boccard, G. Bugnon, C. Denizot, M. Despeisse, A. Feltrin, and C. Ballif, Appl. Phys. Lett. 96, 213504 (2010).

${ }^{12}$ S. Nicolay, M. Despeisse, F.-J. Haug, and C. Ballif, Sol. Energy Mater. Sol. Cells 95, 1031 (2011).

${ }^{13}$ K. Bittkau, M. Schulte, M. Klein, T. Bekcers, and R. Carius, Thin Solid Films 519, 6538 (2011).

${ }^{14}$ K. Bittkau, W. Böttler, M. Ermes, V. Smirnov, and F. Finger, J. Appl. Phys. 111, 083101 (2012).
${ }^{15}$ M. Peters, K. Forberich, C. Battaglia, A. G. Aberle, and B. Bläsi, Proc. SPIE 8438, 84380F (2012).

${ }^{16}$ M. Schulte, K. Bittkau, K. Jäger, M. Ermes, M. Zeman, and B. E. Pieters, Appl. Phys. Lett. 99, 111107 (2011).

${ }^{17}$ K. Jäger, M. Fischer, R. A. C. M. M. van Swaaij, and M. Zeman, J. Appl. Phys. 111, 083108 (2012).

${ }^{18}$ F.-J. Haug, A. Naqavi, and C. Ballif, J. Appl. Phys. 112, 024516 (2012).

${ }^{19}$ D. Dominé, Ph.D. dissertation, Université de Neuchâtel, 2009, p. 107.

${ }^{20}$ C. Battaglia, J. Escarré, K. Söderström, F.-J. Haug, D. Dominé, A. Feltrin, and C. Ballif, Mater. Res. Soc. Symp. Proc. 1245, A03 (2010).

${ }^{21}$ C. K. Carniglia, Opt. Eng. 18, 104 (1979).

${ }^{22}$ M. Boccard, P. Cuony, C. Battaglia, M. Despeisse, and C. Ballif, Phys. Status Solidi (RRL) 4, 326 (2010)

${ }^{23}$ S. Fahr, T. Kirchartz, C. Rockstuhl, and F. Lederer, Opt. Express 19, A865 (2011).

${ }^{24}$ H. Sakai, T. Yoshida, T. Hama, and Y. Ichikawa, Jpn. J. Appl. Phys., Part 129,630 (1990).

${ }^{25}$ M. Despeisse, C. Battaglia, M. Boccard, G. Bugnon, M. Charrière, P. Cuony, S. Hänni, L. Löfgren, F. Meillaud, G. Parascandolo, T. Söderström, and C. Ballif, Phys. Status Solidi A 208, 1863 (2011).

${ }^{26}$ C.-M. Hsu, C. Battaglia, C. Pahud, Z. Ruan, F.-J. Haug, S. Fan, C. Ballif, and Y. Cui, Adv. Energy Mater. 2, 628 (2012).

${ }^{27}$ M. Boccard, T. Söderström, P. Cuony, C. Battaglia, S. Hänni, S. Nicolay, L. Ding, M. Benhaira, G. Bugnon, A. Billet, M. Charrière, F. Meillaud, M. Despeisse, and C. Ballif, IEEE J. Photovoltaics 2, 229 (2012).

${ }^{28}$ M. Boccard, C. Battaglia, S. Hänni, K. Söderström, J. Escarré, S. Nicolay, F. Meillaud, M. Despeisse, and C. Ballif, Nano Lett. 12, 1344 (2012).

${ }^{29}$ H. W. Deckman, C. B. Roxlo, and E. Yablonovitch, Opt. Lett. 8, 491 (1983).

${ }^{30}$ C. Battaglia, L. Erni, J. Escarré, K. Söderström, L. Ding, G. Bugnon, A. Billet, M. Boccard, L. Barraud, S. D. Wolf, F.-J. Haug, M. Despeisse, and C. Ballif, J. Appl. Phys. 109, 114501 (2011).

${ }^{31}$ H. W. Deckman, C. R. Wronski, H. Witzke, and E. Yablonovitch, Appl. Phys. Lett. 42, 968 (1983).

${ }^{32}$ C. Rockstuhl, S. Fahr, K. Bittkau, T. Beckers, R. Carius, F.-J. Haug, T. Söderström, C. Ballif, and F. Lederer, Opt. Express 18, A335 (2010).

${ }^{33}$ P. Cuony, Ph.D. dissertation, EPFL, 2011, p. 26.

${ }^{34}$ M. Boccard, C. Battaglia, F.-J. Haug, M. Despeisse, and C. Ballif, Appl. Phys. Lett. 101, 151105 (2012). 